general problem in $\mathrm{AI}$ that it has no accepted method or style of presenting research and results, in contrast with most experimental sciences. I do not ask for rigid rules and regulations, but AI needs to do a bit better. It is notoriously hard to judge 'good work' in the field and there is a lot of so-so work around - perhaps because there are so many journals to publish in. Scientific progress in any field is aided by agreed criteria for presenting, comparing and contrasting results. If AI improved its methodology, particularly in its applied research, it might encounter fewer brickbats.

In summary, both journals have faults, and I expect them to have a difficult passage. But they both have many virtues and I hope they will find their niche and their audience, despite the stiff competition.

John Fox is in the Advanced Computation Laboratory Unit, Imperial Cancer Research Fund, Lincoln's Inn Fields, London WC2A 3PX, UK.

\section{Theoretical computing}

John A. Campbell

Journal of Logic and Computation. Editorin-chief D. M. Gabbay. Oxford University Press. 6/yr. Europe 1115, elsewhere $\$ 215$ (institutional); Europe 155 , elsewhere $\$ 99$ (personal).

Applicable Algebra in Engineering, Communication and Computing. Managing Editor Jacques Calmet. Springer. 4/yr. DM258, \$161.

Parallel Processing Letters. Editor M. Cosnard. World Scientific. 4/yr. $\$ 180$ (institutional); $\$ 90$ (personal, and institutions from developing countries).

ON the evidence of these journals, theoretical computer science is experiencing a second wave of confidence. The first was marked by the launching of journals open to contributors from all corners of the subject and to people with new corners to suggest. Now computational theory is big enough, secure enough and demonstrably useful enough to justify journals that specialize in particular issues.

Journal of Logic and Computation admits logic-based research with relevance to any area of computer science. In practice, the bias of recent contributions has been towards logic programming and the specification of complex computing systems (for example for concurrent computations) in terms of logical statements about their desired properties. Other connections between logic and computation are possible, particularly in

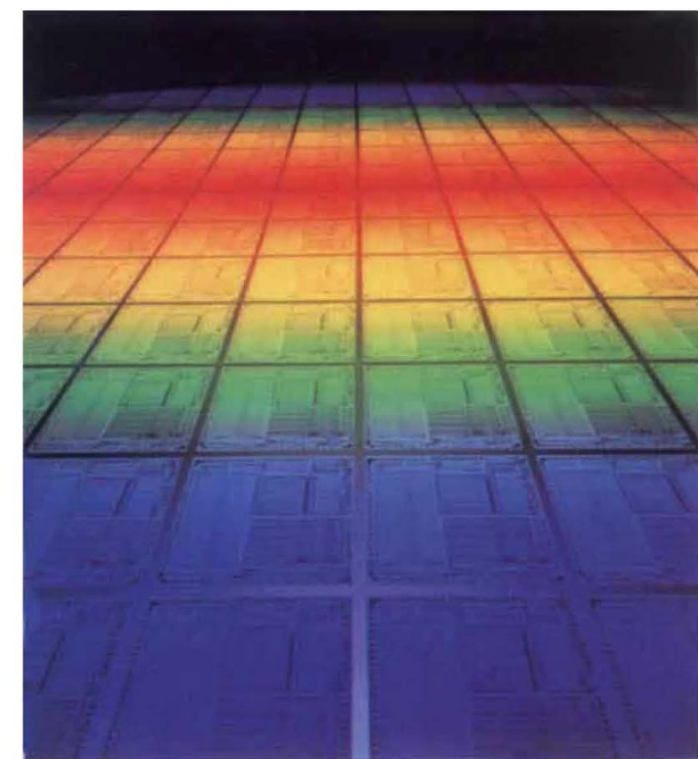

division of interest that ensures that this journal concentrates on the kinds of algebra found in symbolic mathematical computation, coding theory or algebraic geometry. To give an idea of the coverage (which is again for specialists and researchers), the most commonly cited journal in bibliographies of its papers is Mathematics of Computation.

Parallel Processing Letters has two different flavours: it is intended as a 'letters' journal for rapid publication of fairly short papers, and it is (in principle, anyway) driven by the scientific area of computing that is named in its title. The advice to authors contains the intriguing message: "Experimental results are also welcome for publication if they contain an analysis corresponding to an abstract model of computation" (my italics). A possible Parallel processing - macrophotograph of an array of rectangular transputers.

artificial intelligence, but have not been so much in evidence in the journal. An unusual feature is a regular polemical leading article. This may not have been intended to provoke letters to the editor (a category that the journal recognizes), but it deserves to. For specialists, the journal has both value and potential.

As logic tends to turn up within algebra as a topic in computer science degree courses, Applicable Algebra has an even wider coverage: anything algebraic that affects computing and information technology. In practice there is a traditional translation is: "Tell us why you think we theoreticians ought to pay any attention to your results".

Indeed, there is some evidence (well, just a little) in each journal that mathematicians rather than computer scientists are making the running. Subject to this culture warning, I shall be happy to ask my local research librarian to consider subscriptions to all three.

John A. Campbell is in the Department of Computer Science, University College London, Gower Street, London WC1E 6BT, UK.

\title{
From the fundamental to the applied
}

\section{IanA. Walmsley}

International Journal of Nonlinear Optical Physics. Editor-in-chief lam-Choon Khoo. World Scientific. 4/yr. $\$ 348$ (institutional); $\$ 170$ (personal, and institutions from developing countries).

IN today's economic climate, it requires a degree of fortitude for a publisher to launch a highly specialized publication. But World Scientific has done an admirable job in laying the ground-work for this important new journal. The editor and members of the editorial board are all distinguished workers in nonlinear optics (including two of the founding fathers of the field, Professors N. Bloembergen and A. M. Prokhorov). Similarly, contributors to the first few issues comprise an international and well-known group of scientists and engineers.

Papers range from the fundamental to the applied, in roughly equal proportions, and the journal therefore competes directly with several more well-established publications. As a newcomer, it is unlikely to be placed immediately among the journals read directly on publication; for now, it will find a niche as a repository for important and detailed information that needs to be in the literature but which will probably not revolutionize the field. But its status may rise as it becomes more established.

So far, issues have mainly contained lengthy research articles (10-20 pages each). "Rapid communications" (meaning, I presume, short articles) and lengthier review articles will also be published.

The production quality is high, although the layout and style of some of the figures occasionally interrupt the flow of the articles. Subscriptions are cheap and there are no page charges. In short, this is a useful addition to a comprehensive optics research library.

Ian A. Walmsley is in the Institute of Optics, University of Rochester, Rochester, New York 14627, USA. 